\title{
Improved Light Output Power of Chemically Transferred InGaN/GaN Light-Emitting Diodes for Flexible Optoelectronic Applications
}

\author{
Ho-Jun Lee, ${ }^{1}$ Jung-Wook Min, ${ }^{2}$ Kye-Jin Lee, ${ }^{3}$ Kwang-Yong Choi, ${ }^{3}$ Jung-Hyun Eum, ${ }^{3}$ \\ Dong-Kun Lee, ${ }^{4}$ and Si-Young Bae ${ }^{1}$ \\ ${ }^{1}$ Department of Electrical Engineering and Computer Science, Nagoya University, Nagoya, Aichi 464-8603, Japan \\ ${ }^{2}$ Department of Physics and Photon Science, Gwangju Institute of Science and Technology (GIST), Gwangju 500-712, Republic of Korea \\ ${ }^{3}$ HPC Advanced Development Team, LG Innotek, Paju, Gyeonggi-do 413-901, Republic of Korea \\ ${ }^{4}$ Wafer Characteristics Research Team, LG Siltron, Gumi, Gyeongsangbuk-do 730-724, Republic of Korea \\ Correspondence should be addressed to Si-Young Bae; siyoubae@gmail.com
}

Received 14 August 2015; Accepted 27 September 2015

Academic Editor: Kamal Alameh

Copyright (C) 2015 Ho-Jun Lee et al. This is an open access article distributed under the Creative Commons Attribution License, which permits unrestricted use, distribution, and reproduction in any medium, provided the original work is properly cited.

\begin{abstract}
Recent needs of semiconductor lighting sources have pursued diverse functionalities such as flexibility and transparency under high quantum efficiency. Inorganic/organic hybrid light-emitting diodes (LEDs) are one way to meet these requirements. Here, we report on flexible III-nitride-based LEDs and the improvement of their electrical and optical properties. To realize high light emission power and stable current operation, high-quality epitaxy and elaborate chip processing were performed. The fabricated flexible LEDs showed over threefold optical output power compared to normal LEDs on Si and had comparable forward voltage and series resistances.
\end{abstract}

\section{Introduction}

Inorganic/organic hybrid processing technology has been extensively exploited for semiconductor-based optoelectronic applications [1]. In particular, hybrid light-emitting devices (LEDs) have been of interest, since inorganic and organic LEDs have many similarities and are competing as ultimate lighting sources. For example, organic light-emitting diodes (OLEDs) are superior to inorganic LEDs in terms of their large emission area, low cost, transparency, and flexible operation [2]. However, OLEDs have many drawbacks that must be solved: low brightness, low quantum efficiency, short lifetime, and low stability in high temperature and humidity [3-5]. The compatibility of both systems can be found in metallization, etching-based fabrication, and attachable/detachable processing. In particular, inorganic LEDs can be integrated on organic films, thereby demonstrating flexible/stretchable/transparent LEDs by keeping their inherent merits. Several groups have recently demonstrated flexible LEDs using inorganic III-V LED chip transfer to flexible plastic substrates, such as polyimide and polyethylene terephthalate (PET) $[6,7]$. Furthermore, the chip transfer approach offers the chance to demonstrate implantable biomedical applications as well as wearable displays [7-9].

To date, although conceptual demonstrations have been performed, key factors are still required to reach commercial utilization: (1) high-quality epitaxy of III-V inorganic LEDs; (2) reduction of quality degradation during chemical processes including bonding and etching processes; (3) precise alignment of inorganic/organic materials; and (4) good Ohmic metallization of transferred chips. In particular, substrates for epitaxy must be easily removed in chemical solutions to satisfy the second requirement noted above. Hence, this study has dealt with III-nitride LEDs grown on Si substrates. Although there have been several issues with $\mathrm{GaN}$ epitaxy on $\mathrm{Si}$, such as stress management and dislocation reduction, high-quality GaN epilayers have been optimized and used [10]. In addition, to acquire high light emission with the good Ohmic metallization mentioned in the fourth requirement above, $\mathrm{Si}$ substrates can be easily removed and 


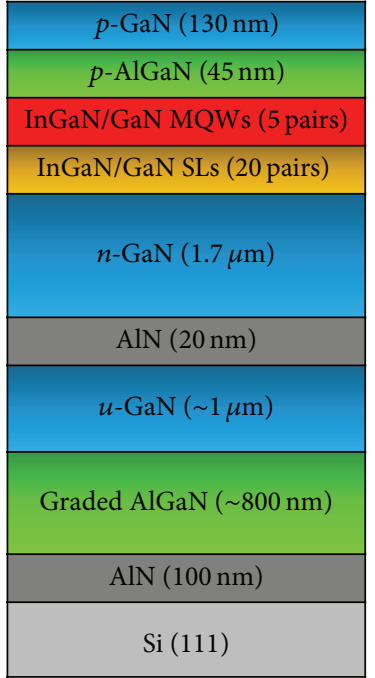

(a)

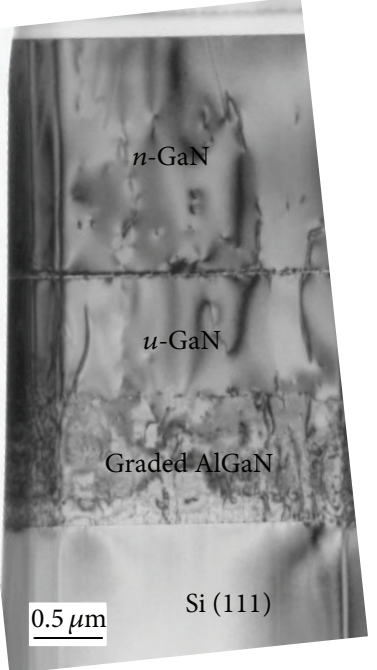

(b)

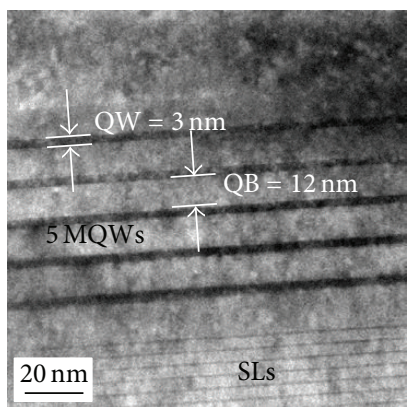

(c)

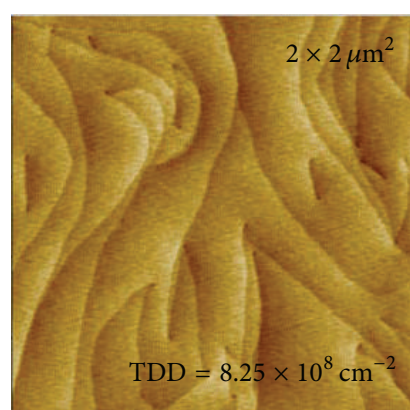

(d)

FIGURE 1: (a) Schematic of grown epitaxial LED structures, cross-sectional TEM images for (b) $n$-GaN template regions and (c) InGaN/GaN MQW regions, and (d) AFM images of LED epilayer $\left(2 \times 2 \mu \mathrm{m}^{2}\right)$.

highly reflective metals can be substituted for the mother layers, thereby eliminating strong visible light absorption [10]. Therefore, in this paper, we demonstrate the improved electrical and optical properties of chemically transferred InGaN/GaN LEDs for flexible optoelectronic applications.

\section{Experimental Method}

GaN on Si LED structures were grown on 2-inch Si (111) substrates by metalorganic chemical vapor phase deposition (MOCVD, AIXTRON Aix2000). Trimethylgallium (TMGa), trimethylaluminum (TMAl), trimethylindium (TMIn), and ammonia $\left(\mathrm{NH}_{3}\right)$ were used as precursors for the III and $\mathrm{V}$ sources. Disilane $\left(\mathrm{Si}_{2} \mathrm{H}_{6}\right)$ and cyclopentadienyl magnesium $\left(\mathrm{Cp}_{2} \mathrm{Mg}\right)$ were used as the sources for the n-type and $\mathrm{p}$ type dopants. First, Si substrates were heated up to $1080^{\circ} \mathrm{C}$ for 10 min under $\mathrm{H}_{2}$ ambient. To protect the Si surface from melt-back etching, the TMAl source was introduced for $10 \mathrm{~s}$. Subsequently, a $100 \mathrm{~nm}$ thick AlN layer was grown at $1020^{\circ} \mathrm{C}$. For the graded AlGaN buffer layers, the $\mathrm{Al}$ compositions were controlled to change from $75 \%$ to $5 \%$. The temperatures for $u$-GaN and upper AlN were maintained at $1050^{\circ} \mathrm{C}$ and $780^{\circ} \mathrm{C}$, respectively. An n-type doped $\mathrm{GaN}$ was grown at $1050^{\circ} \mathrm{C}$, and the n-type doping concentration was kept at $\sim 5 \times 10^{18} \mathrm{~cm}^{-3}$. As for the active layers, 20 periods of $\mathrm{In}_{0.05} \mathrm{Ga}_{0.95} \mathrm{~N} / \mathrm{GaN}(2 \mathrm{~nm} / 2 \mathrm{~nm})$ were grown at $800^{\circ} \mathrm{C}$; five periods of $\mathrm{In}_{0.13} \mathrm{Ga}_{0.87} \mathrm{~N} / \mathrm{GaN}(3 \mathrm{~nm} / 12 \mathrm{~nm}$ ) multiple quantum wells (MQWs) were grown at the same $760^{\circ} \mathrm{C}$. Finally, $p-\mathrm{Al}_{0.21} \mathrm{Ga}_{0.79} \mathrm{~N}$ as an electron blocking layer (EBL) and $p$ $\mathrm{GaN}$ were grown at the same $960^{\circ} \mathrm{C}$, where the p-type doping concentrations of $p-\mathrm{AlGaN}$ and $p-\mathrm{GaN}$ were maintained at $\sim 1$ $\times 10^{20} \mathrm{~cm}^{-3}$.

As for the material/optical/electrical characterization, the surface morphology was observed using a scanning electron microscope (SEM, Hitachi S-4700) and an atomic force microscope (AFM, Park Systems XE15). The crystal quality and detailed microstructures of $\mathrm{GaN}$ were characterized using a high resolution transmission electron microscope (HRTEM, FEI Tecnai G2F30 twin system). The electrical and optical properties of the fabricated devices were characterized by LED chip measurement (EPS1000 Ecopia system) with an integrating sphere. Here, the single LED chip had a size of $\sim 0.25 \mathrm{~mm}^{2}$. For convenience and simplicity, detailed fabrications are described in the next section.

\section{Results and Discussion}

Prior to the fabrication of the flexible LEDs, the epitaxial qualities of the LED structures were investigated. A schematic of the grown epitaxial LED structures is presented in Figure 1(a). It consisted of a $100 \mathrm{~nm} \mathrm{AlN}$, an $800 \mathrm{~nm}$ graded AlGaN layer, a $1 \mu \mathrm{m}$ undoped GaN layer, a $20 \mathrm{~nm}$ AlN layer, a $1.7 \mu \mathrm{m}$ Si-doped n-type GaN layer, 20 periods of InGaN/GaN superlattices (SLs), five periods of InGaN/GaN MQWs, a $90 \mathrm{~nm} \mathrm{Mg-doped} \mathrm{p-type} \mathrm{AlGaN} \mathrm{layer,} \mathrm{and} \mathrm{a} 130 \mathrm{~nm} \mathrm{Mg-}$ doped p-type GaN layer. Here, the graded AlGaN layers were aimed at releasing the biaxial strain between $\mathrm{Si}$ and $\mathrm{GaN}$, thereby avoiding the generations of cracks during the epitaxy cooling process [11]. Figure 1(b) shows the cross-sectional TEM images of the $n$-GaN template regions. Typically, a high density of defects was found at the GaN/Si interfaces. By growing the graded AlGaN layer and the upper AlN layer, dislocations were noticeably reduced. Here, the threading dislocation density was found to be $8.25 \times 10^{-8} \mathrm{~cm}^{-2}$, comparable to that of a typical $\mathrm{GaN}$ epilayer on common $c$-plane sapphire substrates [12]. Figure 1(c) displays the cross-sectional TEM images of the MQW and SL regions. For the uniform blue emission with a target wavelength $(\lambda=450 \mathrm{~nm})$, interfaces at MQWs and SLs were clearly managed. We could hardly observe thickness variation or indium segregation features 


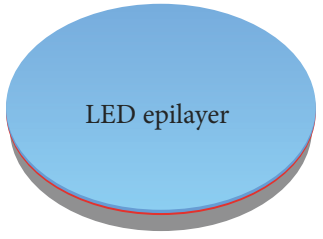

Epi. on $\mathrm{Si}$

(a)

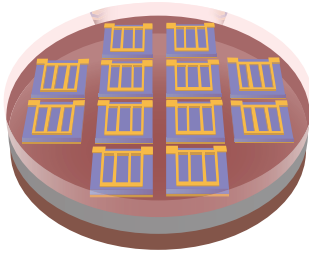

PDMS coating

(e)

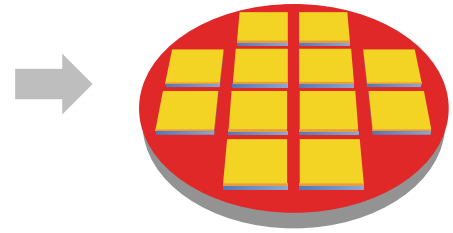

Chip process

(b)

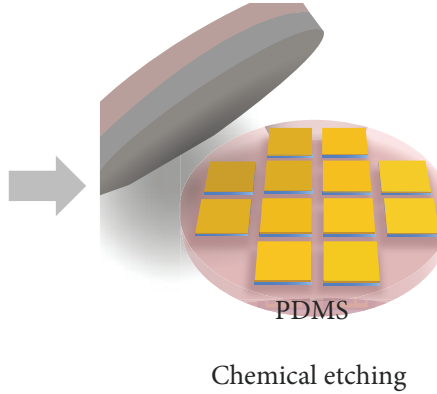

(f)
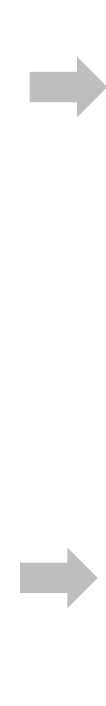

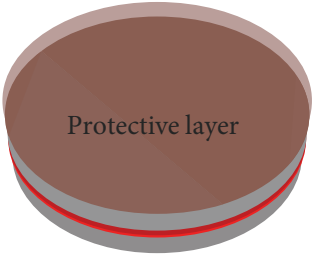

Wafer bonding

(c)

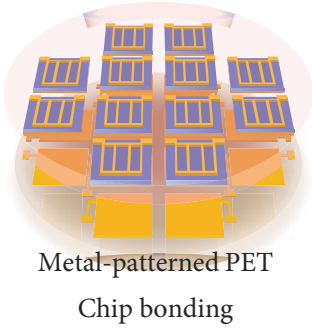

(g)

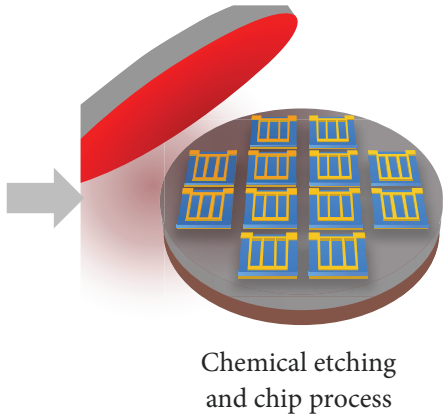

(d)

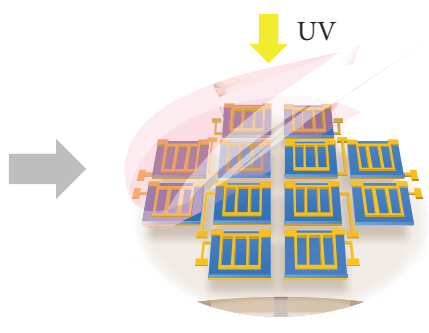

LEDs on PET

(h)

Figure 2: Schematic of flexible LED procedures. (a) Epistructures, (b) chip fabrication on $p$-GaN side, (c) wafer bonding to supporter and polymer covering, (d) mother Si substrate removal and chip fabrication on $n$-GaN side, (e) PDMS deposition and curing, (f) supporting substrate removal, (g) LED chip transfer to metal-patterned PET films, and (h) PDMS removal by UV exposure and flexible LEDs on PET films.

in the InGaN layer, which critically affect light intensity degradation as well as spectrum broadening [13, 14]. The thicknesses of the quantum well (QW) and the quantum barrier (QB) were uniformly kept at $3 \mathrm{~nm}$ and $12 \mathrm{~nm}$, respectively. The SLs were aimed at releasing the biaxial strain at the InGaN/GaN interfaces of the MQWs, thus increasing indium incorporation as well as internal quantum efficiency [15]. To determine the epitaxial quality and surface morphology of the underlying $n$-GaN layer, an AFM measurement was taken for a $2 \times 2 \mu \mathrm{m}^{2}$ area (Figure $1(\mathrm{~d})$ ). Pits were hardly observed, and smooth surface with terrace-like atomic features was observed. Hence, the grown epitaxial structures were highly controlled to be fabricated for the next chemical transfer step.

Once the LED epilayers were grown, inorganic chip fabrication and transfer were performed. Figure 2 presents a schematic of the fabrication process for flexible LEDs. First, metallization was performed on the p-type doped GaN side after mesa etching by an inductively coupled plasma (ICP) etcher (Figures 2(a) and 2(b)). Here, ITO, Ag, and $\mathrm{Au} / \mathrm{Sn}$ metal layers were deposited using an e-beam evaporator. This metallization process aims to acquire good Ohmic properties, high reflectivity, and suitable adhesion onto the supporting substrate [16]. After bonding a chip-on-substrate to another supporter, the enamel polymer was covered on the supporter surface (Figure 2(c)). Then, the mother Si substrate was chemically removed by HNA (HF : Nitric: Acetic) wet solution while the supporter remained chemically durable owing to the protective polymer. Subsequently, chip fabrication on the n-doped GaN side was performed after etching the remaining AlGaN and $u$-GaN layers with ICP for the vertical current conduction (Figure 2(d)). To ensure the high lightextraction efficiency of LEDs, $\mathrm{N}$-face $n$-GaN was chemically treated using a $\mathrm{KOH}$ solution to form random pyramidal nanostructures. Then, the polydimethylsiloxane (PDMS) was attached onto the fabricated chips to facilitate the handling of the isolated LED chips (Figure 2(e)). The supporting substrate was subsequently removed by dipping it in HNA wet solution, and the $p$-electrode side was exposed again (Figure 2(f)). The separated LED chips were aligned and attached on metal-grid patterned PET films by van der Waals force (Figure 2(g)). Finally, the PDMS films were detached from the surface by UV exposure, and the LEDs were ready for the injecting current (Figure 2(h)).

Figure 3 provides real images of the particular chip fabrication process. First, one example in which the $\mathrm{Si}$ supporter was properly attached by a wafer bonding process is shown in Figure 3(a). We also confirmed the exact aligned features by the enlarged camera image, as seen in the red rectangle. Normally, GaN/Si epitaxy suffers from crack generation due to the large thermal mismatch between $\mathrm{GaN}$ and Si and the resulting strain mismatch [17]. In order to reduce additional crack generation due to the additional pressure from the wafer bonding process, we have tried to use optimized eutectic melting temperature as well as pressure. Then, most of the LED chips were successfully transferred to the supporter except for the edge regions of $\sim 2 \mathrm{~mm}$. Although 

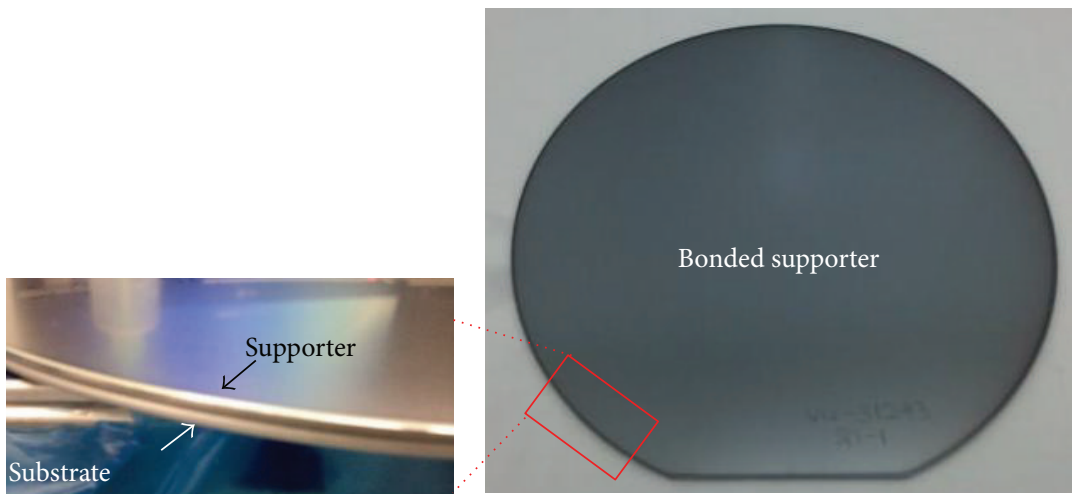

(a)

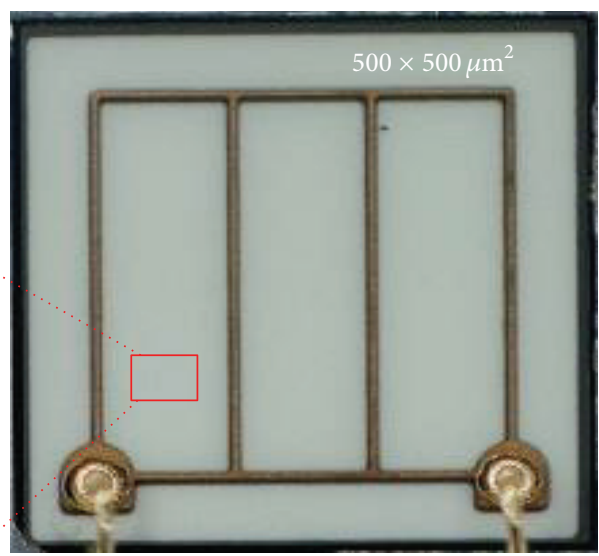

(c)

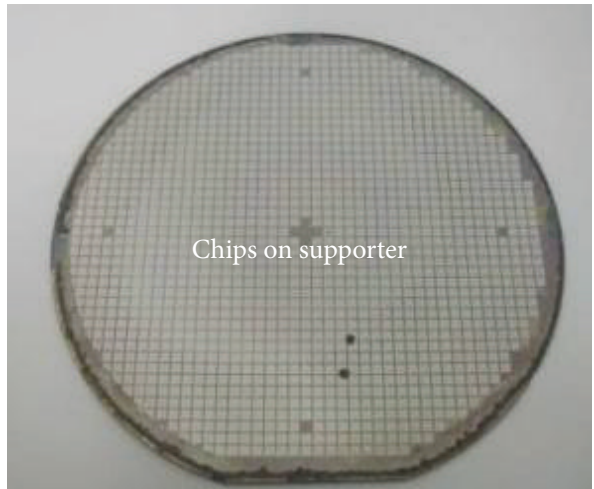

(b)

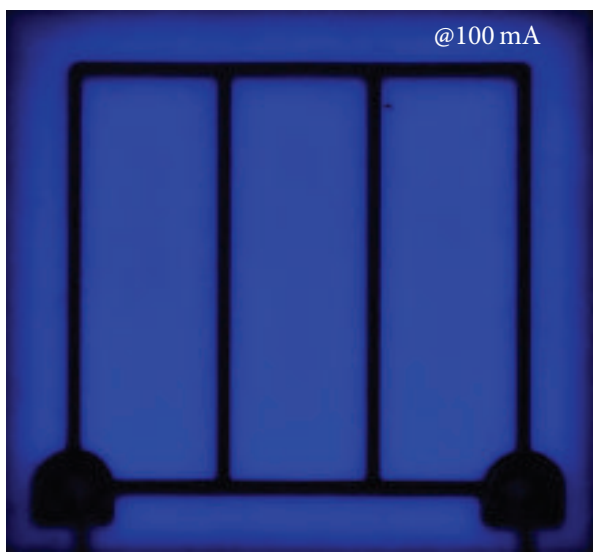

(d)

FIGURE 3: Camera image of (a) bonded Si supporter and (b) fabricated LED chips on supporter after mother substrate removal. (c) Optical microscope image of single chip on $n$-electrode side and (d) corresponding image under current injection of $100 \mathrm{~mA}$. The insets of (a) and (c) are enlarged camera image at edge of bonded wafers and SEM image of $\mathrm{N}$-face $n$-GaN region after KOH etching, respectively.

the partial edges were not available due to the bonding process, the production yield $(\sim 80 \%)$ shown in this study is quite encouraging, since the edges of common epitaxy also cannot maintain uniformity in terms of wavelength and light output power and are thereby excluded for commercial use [18]. Figure 3(c) shows a detailed feature of a fabricated chip (500 $\times 500 \mu \mathrm{m}^{2}$ ) using an optical microscope image. By enlarging a particular region (red rectangle) of the chip surface between metal grids, we observed nanopyramid structures of SEM images formed by $\mathrm{KOH}$ wet etching. The formation of $\mathrm{GaN}$ nanostructures can significantly improve the light-extraction efficiency by suppressing the Fresnel internal reflection of LED layers [19]. In these LED chips, we observed highly uniform light emission, as shown in Figure 3(d). Under the current injection of $100 \mathrm{~mA}$, pure blue emission was found without any of the parasitic current crowding commonly found on lateral LEDs [20].

Figure 4(a) displays the light emission images of flexible LEDs resulting from changing the current injection from $10 \mathrm{~mA}$ to $100 \mathrm{~mA}$. Brighter emissions were found when higher current injections were applied. Here, the relatively weak emission at $20 \mathrm{~mA}$ compared to conventional LEDs might be attributed to the low current density due to the large chip size $\left(500 \times 500 \mu \mathrm{m}^{2}\right)$. Considering the amount of current density on this chip, we determined the forward voltage to be $60 \mathrm{~mA}$ (i.e., $2.4 \mathrm{~A} / \mathrm{cm}^{2}$ ) in this study. Figure 4(b) presents the voltage-current $(V-I)$ characteristics of normal LEDs on $\mathrm{Si}$ (N-LEDs) and flexible LEDs (F-LEDs). To ensure fair comparison with F-LEDs, lateral type N-LEDs were fabricated on mother Si substrates with a chip size of $350 \times$ $700 \mu \mathrm{m}^{2}$ (i.e., almost the same chip size as the F-LEDs). For the fabrication of the lateral chip, a $150 \mathrm{~nm}$ thick ITO was used as the current spreading layer of $p$-GaN, and $n$-electrodes (Ti/Al/Au alloys) were deposited on etched $n-\mathrm{GaN}$ around the LED mesa region over the Si epilayer. The electrodes were connected to external pads by Au wire bonding to be measured in the integrating sphere. The obtained forward voltages were $3.84 \mathrm{~V}$ and $3.90 \mathrm{~V}$ for N-LEDs and F-LEDs, respectively, while almost similar series resistances were found $(\sim 5.25 \Omega)$ for both LEDs. It is speculated that the slightly higher forward voltage of the F-LEDs was caused by the thermal instability of the PET films. However, note that the degradation was not severe and the electrical properties were quite similar to those of the N-LEDs. This indicates that the F-LEDs were far superior to the other transferred flexible LEDs reported elsewhere [6,7]. The light output 

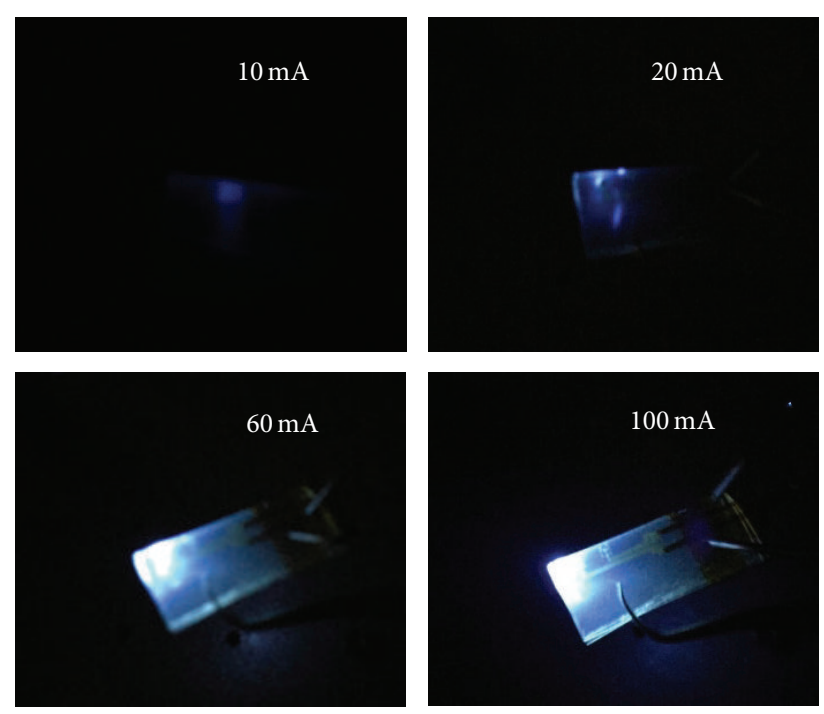

(a)

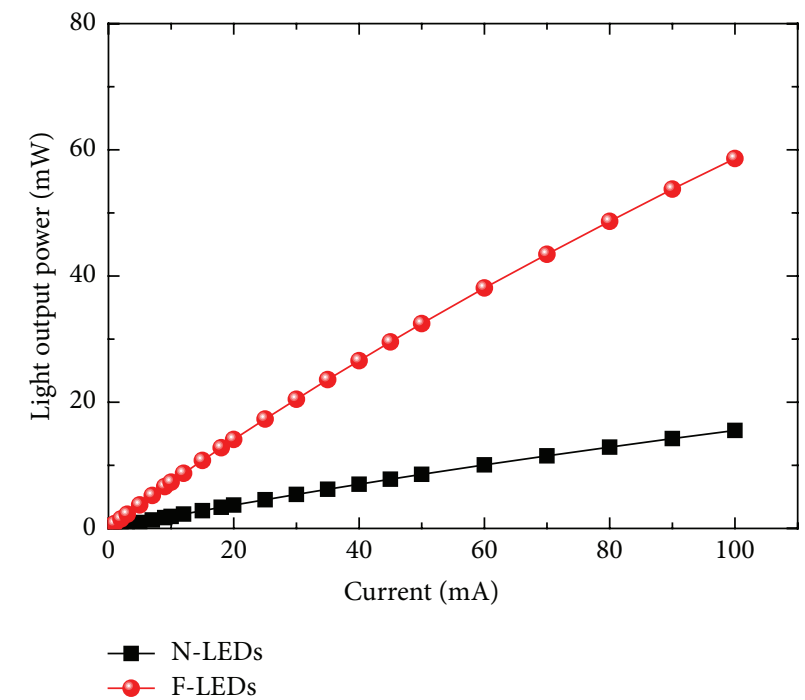

(c)

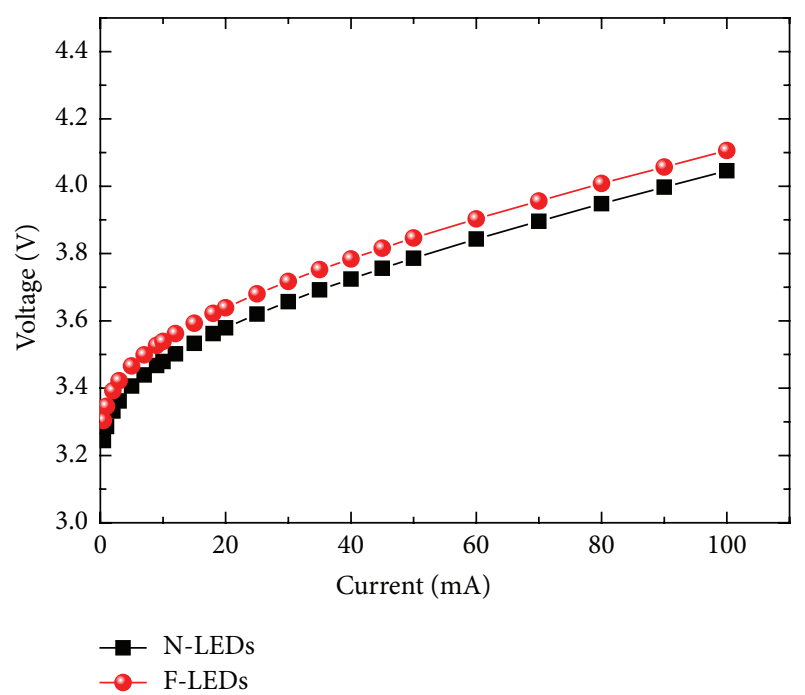

(b)

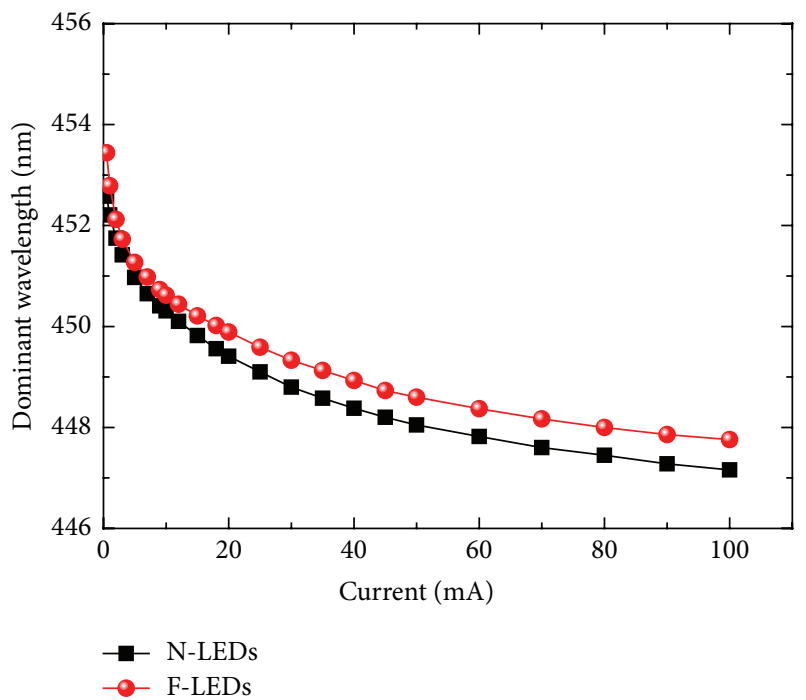

(d)

FIGURE 4: (a) Light emission of F-LEDs by changing current injection from $10 \mathrm{~mA}$ to $100 \mathrm{~mA}$, (b) $V$ - $I$ characteristics, (c) $L-I$ characteristics, and (d) dominant wavelength variation as a function of current injection.

power of the F-LEDs was also significantly enhanced by $378 \%$ at $100 \mathrm{~mA}$ compared to that of the N-LEDs in Figure 4(c). Here, the samples were measured in the integrating sphere to collect all light emissions. Hence, we believe that the huge enhancement of the optical output power for the F-LEDs was attributed to the removal of the Si substrate, which absorbs most of the visible spectrum. In addition, Figure 4(d) shows the dominant wavelength as a function of injection current. For both cases, the variation was distributed within $5 \mathrm{~nm}$, indicating highly stable wavelength variation for both LEDs.

\section{Conclusion}

In this work, we reported on flexible LEDs transferred from III-nitride LEDs on Si substrates. For the GaN on the Si epitaxy, we grew high-quality LEDs that had few defects and were crack-free. To eliminate Si substrates and prevent physical degradation, Si substrates were easily removed from the wet solution. After confirming the uniform light emission on the LEDs on Si (N-LEDs), the III-nitride LEDs were transferred to metal-patterned PET substrates using an adhesive PDMS layer. The fabricated flexible LEDs (FLEDs) not only showed comparable electrical properties compared to the N-LEDs, but also had much improved optical output power (over threefold). Furthermore, the FLEDs were properly operated under a high current injection level at $100 \mathrm{~mA}$. Therefore, we believe that this method can contribute to improving the electrical and optical properties of hybrid flexible LEDs, and it might advance the commercialization of flexible/wearable/transparent displays with inorganic/organic hybrid integration. 


\section{Conflict of Interests}

The authors declare that there is no conflict of interests regarding the publication of this paper.

\section{References}

[1] R. Houbertz, G. Domann, C. Cronauer et al., "Inorganicorganic hybrid materials for application in optical devices," Thin Solid Films, vol. 442, no. 1-2, pp. 194-200, 2003.

[2] N. T. Kalyani and S. J. Dhoble, "Organic light emitting diodes: energy saving lighting technology-a review," Renewable and Sustainable Energy Reviews, vol. 16, no. 5, pp. 2696-2723, 2012.

[3] T. Nakayama, K. Hiyama, K. Furukawa, and H. Ohtani, "Development of a phosphorescent white OLED with extremely high power efficiency and long lifetime," Journal of the Society for Information Display, vol. 16, no. 2, pp. 231-236, 2008.

[4] F. A. Ponce and D. P. Bour, "Nitride-based semiconductors for blue and green light-emitting devices," Nature, vol. 386, no. 6623, pp. 351-359, 1997.

[5] D. A. Gaul and W. S. Rees Jr., "True blue inorganic optoelectronic devices," Advanced Materials, vol. 12, no. 13, pp. 935-946, 2000.

[6] R.-H. Kim, S. Kim, Y. M. Song et al., "Flexible vertical light emitting diodes," Small, vol. 8, no. 20, pp. 3123-3128, 2012.

[7] S. Y. Lee, K.-I. Park, C. Huh et al., "Water-resistant flexible GaN LED on a liquid crystal polymer substrate for implantable biomedical applications," Nano Energy, vol. 1, no. 1, pp. 145-151, 2012.

[8] S.-I. Park, A.-P. Le, J. Wu, Y. Huang, X. Li, and J. A. Rogers, "Light emission characteristics and mechanics of foldable inorganic light-emitting diodes," Advanced Materials, vol. 22, no. 28, pp. 3062-3066, 2010.

[9] S.-I. Park, Y. Xiong, R.-H. Kim et al., "Printed assemblies of inorganic light-emitting diodes for deformable and semitransparent displays," Science, vol. 325, no. 5943, pp. 977-981, 2009.

[10] D. Zhu, D. J. Wallis, and C. J. Humphreys, "Prospects of IIInitride optoelectronics grown on Si," Reports on Progress in Physics, vol. 76, no. 10, Article ID 106501, 2013.

[11] A. Able, W. Wegscheider, K. Engl, and J. Zweck, "Growth of crack-free GaN on $\mathrm{Si}\left(\begin{array}{lll}1 & 1 & 1\end{array}\right)$ with graded AlGaN buffer layers," Journal of Crystal Growth, vol. 276, no. 3-4, pp. 415-418, 2005.

[12] D. Kapolnek, X. H. Wu, B. Heying et al., "Structural evolution in epitaxial metalorganic chemical vapor deposition grown $\mathrm{GaN}$ films on sapphire," Applied Physics Letters, vol. 67, no. 11, pp. 1541-1543, 1995.

[13] Y. Narukawa, Y. Kawakami, S. Fujita, S. Fujita, and S. Nakamura, "Recombination dynamics of localized excitons in $\mathrm{In}_{0.20} \mathrm{Ga}_{0.80} \mathrm{~N}-\mathrm{In}_{0.05} \mathrm{Ga}_{0.95} \mathrm{~N}$ multiple quantum wells," Physical Review B, vol. 55, no. 4, pp. R1938-R1941, 1997.

[14] G. Pozina, J. P. Bergman, B. Monemar, T. Takeuchi, H. Amano, and I. Akasaki, "Origin of multiple peak photoluminescence in InGaN/GaN multiple quantum wells," Journal of Applied Physics, vol. 88, no. 5, pp. 2677-2681, 2000.

[15] E. Feltin, B. Beaumont, M. Laügt et al., "Stress control in GaN grown on silicon (111) by metalorganic vapor phase epitaxy," Applied Physics Letters, vol. 79, no. 20, pp. 3230-3232, 2001.

[16] T. Jeong, K. H. Kim, H. H. Lee et al., "Enhanced light output power of GaN-based vertical light-emitting diodes by using highly reflective ITO-Ag-Pt reflectors," IEEE Photonics Technology Letters, vol. 20, no. 23, pp. 1932-1934, 2008.
[17] A. Dadgar, J. Bläsing, A. Diez, A. Alam, M. Heuken, and A. Krost, "Metalorganic chemical vapor phase epitaxy of crackfree $\mathrm{GaN}$ on $\mathrm{Si}(111)$ exceeding $1 \mu \mathrm{m}$ in thickness," Japanese Journal of Applied Physics, vol. 39, no. 11, pp. L1183-L1185, 2000.

[18] A. Dadgar, C. Hums, A. Diez, J. Bläsing, and A. Krost, "Growth of blue GaN LED structures on 150-mm Si(1 11 )," Journal of Crystal Growth, vol. 297, no. 2, pp. 279-282, 2006.

[19] T. Fujii, Y. Gao, R. Sharma, E. L. Hu, S. P. DenBaars, and S. Nakamura, "Increase in the extraction efficiency of GaN-based light-emitting diodes via surface roughening," Applied Physics Letters, vol. 84, no. 6, pp. 855-857, 2004.

[20] X. Quo and E. F. Schubert, "Current crowding in GaN/InGaN light emitting diodes on insulating substrates," Journal of Applied Physics, vol. 90, no. 8, pp. 4191-4195, 2001. 

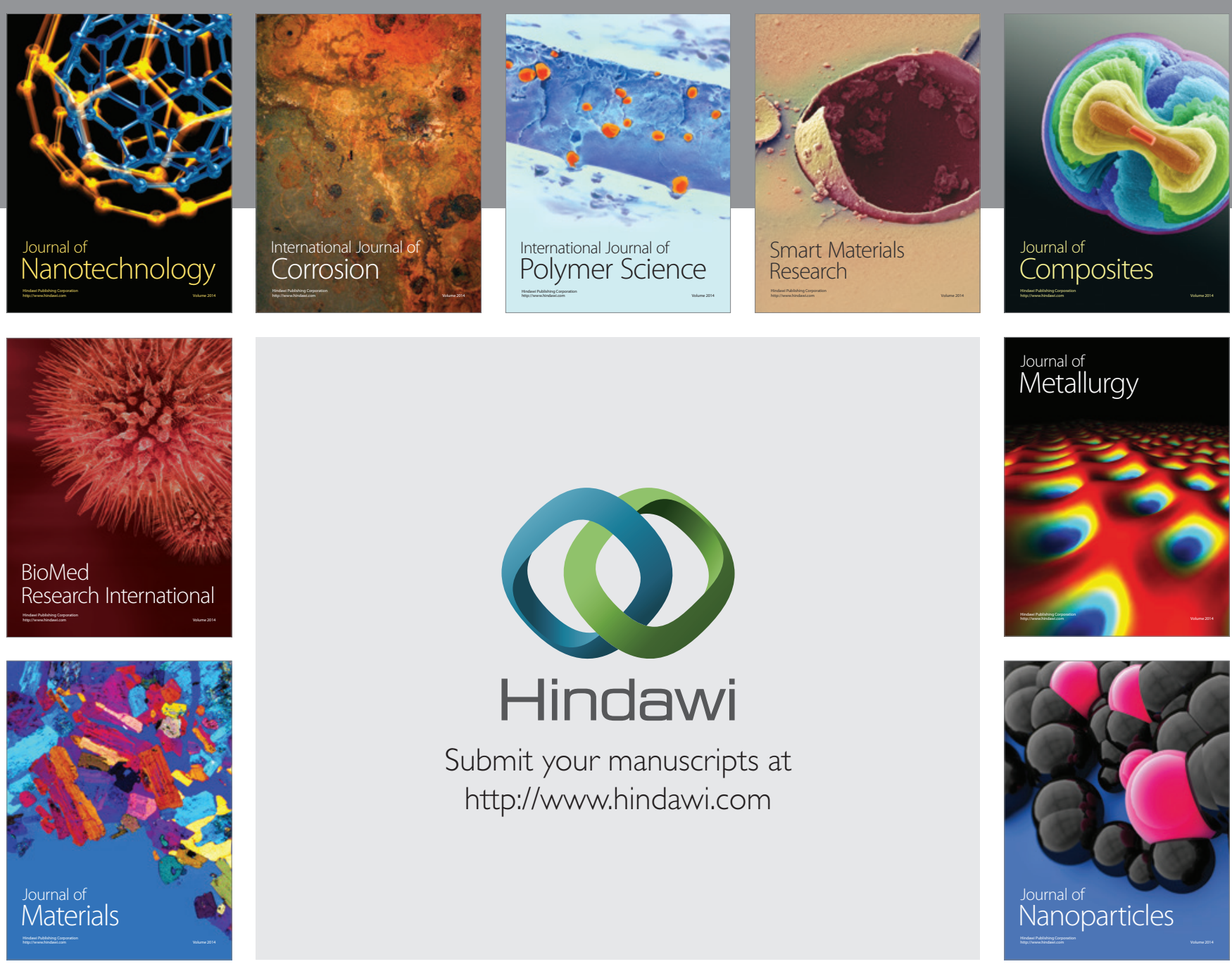

Submit your manuscripts at http://www.hindawi.com
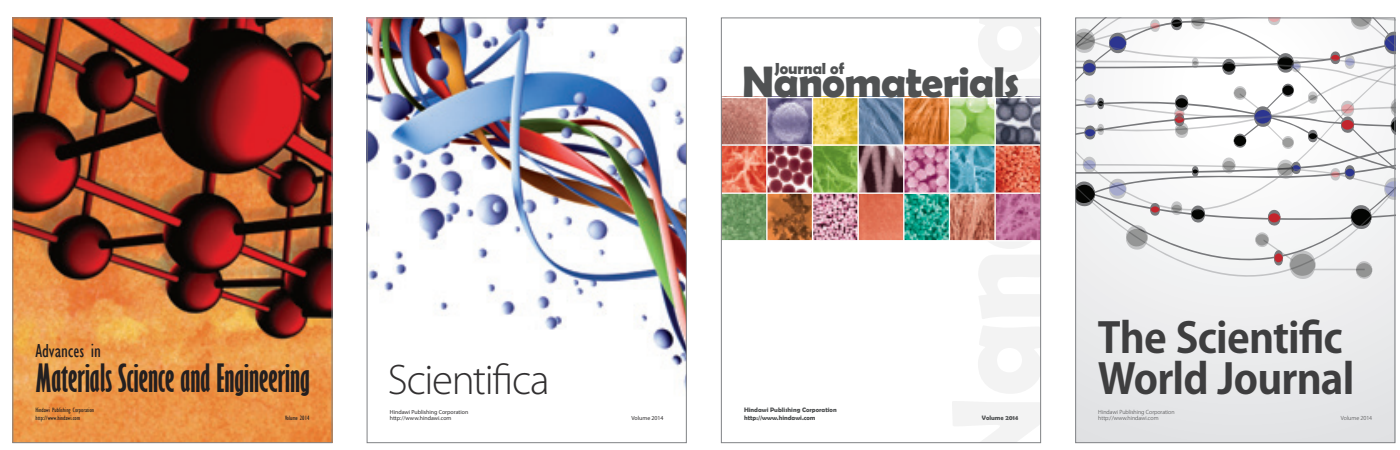

\section{The Scientific World Journal}
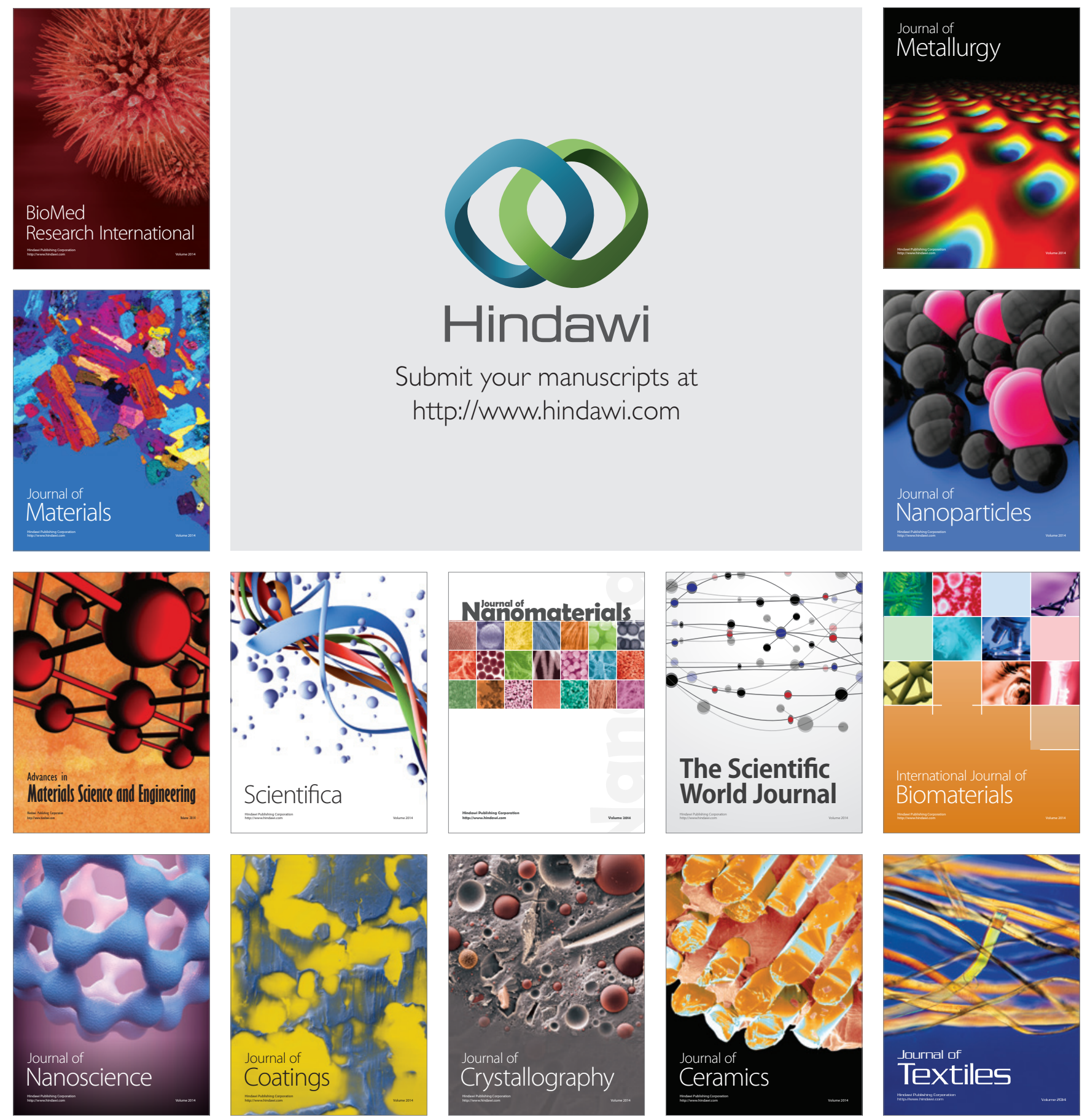